\title{
Engine Integration Based on Multi-Disciplinary Optimisation Technique
}

\author{
Danil Haar*, Joël Brezillon ${ }^{\#}$ \\ DLR Institute of Aerodynamics and Flow Technology \\ Lilienthalplatz 7, 38108 Braunschweig, Germany \\ danil.haar@dlr.de*,joel.brezillon@dlr.de
}

\begin{abstract}
The paper presents the work related to the engine integration of a Rear Fuselage Mounted Engine configuration in the frame of the DLR/Onera project MDOrmec („Multi-Disciplinary Optimisation of Rear-fuselage Mounted Engine Configuration“). The developed multidisciplinary optimization process is based on a multi-level fidelity approach, where the aerodynamics is simulated using CFD methods while the weight and the handling qualities are assessed using preliminary design approach. Finally, the results of the optimisation are presented and discussed in detail.
\end{abstract}

Keywords: Engine Integration, Multidisciplinary Optimization, Aerodynamics, Weight, Flight Mechanics

$\begin{array}{ll}\text { Nomenclature } \\ \mathrm{a} / \mathrm{c} & \text { aircraft } \\ \mathrm{c} & \text { speed of sound } \\ \mathrm{c}_{\mathrm{MAC}} & \text { main cord length } \\ \mathrm{C}_{\mathrm{M}} & \text { pitching moment coefficient } \\ \mathrm{S} & \text { area } \\ \mathrm{SM} & \text { static margin } \\ \mathrm{V} & \text { speed } \\ \mathrm{W} & \text { mass } \\ \text { MTOW } & \text { Maximum Take-Off Weight }\end{array}$

$\begin{array}{ll}\text { sup and subscripts } \\ \text { a/c } & \text { Aircraft } \\ \text { C } & \text { Control } \\ \text { D } & \text { Drag } \\ \text { E } & \text { Engine } \\ \text { H } & \text { horizontal tail plane } \\ \text { M } & \text { Minimal } \\ \text { S } & \text { Stability } \\ \text { V } & \text { vertical tail plane } \\ \text { W } & \text { Wing }\end{array}$

\section{Introduction}

The engine integration is a very important aspect for the aircraft design since the interferences caused by the nacelle and the pylon may badly influence the aerodynamic performance of an aircraft. The detailed analysis of the airframe/propulsion integration done at DLR and ONERA in the 90's [1] shows the substantial interference effects due to the installation of the nacelles under the wing causing the increase of the total drag at constant lift. More recently, similar work were conducted in the frame of the EU-projects ROSAS and NACRE on rear fuselage mounted engine (RFME) configuration. However, in these studies the aerodynamic optimisations were conducted without multiple disciplines coupling [2].

In the present study, the work focuses on the development of a multi-disciplinary process for the optimal integration of the engine on the rear fuselage. The final target of this MDO process is to allow the design of a RFME configuration with the best aircraft performance and the proper engine positions for the best shielding of noise sources by the airframe structure (wing, fuselage, tail). However, the engine represents about $10 \%$ of the MTOW and a change of its position will impact the aircraft centre of gravity $(\mathrm{CG})$ and thus the static margin of the aircraft during the optimisation. Since it is an important criterion from the overall aircraft design point of view, the static margin and more generally the handling qualities, are also integrated into the MDO process.

After presenting the optimisation scenario, the paper presents the first step achieved on the set-up of the MDO process and gives insight into the aircraft performance and the han- 
dling qualities modules. The resulting MDO process is then used to find the optimal engine position which improves the aircraft performance for given handling qualities.

\section{Optimisation Scenario}

The developed MDO process has to be assessed to find the optimal engine position of a regional jet airliner featuring rear fuselage mounted engine and $\mathrm{H}$-shaped tail planes. The reference configuration for the aircraft mission, the handling qualities and shapes (fuselage, wing, engine, pylon, VTP and HTP) is the Fairchild Dornier Do 728 aircraft. The RFME baseline geometry is designed using preliminary design tools. It is a low wing configuration with two engines attached at the rear fuselage end and the H-shaped tail planes, and is derived from the jet airliner Dornier Do 728 in order to provide the best jet noise shielding. The noise aspect is not considered in this work.

\subsection{Objectives}

The aircraft performance selected is the Breguet range [3] which expresses the compromise between the aerodynamic performance of the aircraft and its weight. The formula here retained is

$$
R=c \cdot M_{\infty} \cdot \frac{C_{L}}{C_{D}} \cdot \frac{1}{C_{S F C}} \ln \left(\frac{W_{\text {StartofCruise }}}{W_{\text {EndofCruise }}}\right)
$$

where $M_{\infty}$ is the cruise Mach number, $C_{S F C}$ is the thrust specific fuel consumption and $C_{L}$ (resp. $\mathrm{C}_{\mathrm{D}}$ ) is the lift (resp. the drag) at start of cruise. The aerodynamic forces have to be evaluated on the full configuration in trimmed condition and for a given lift condition, corresponding at the start of cruise.

From the structure side, the MTOW is kept constant during the optimisation. The weight at the end of cruise is obtained by assuming that all fuel is used:

$$
W_{\text {Endoffruise }}=W_{\text {Takeoff }}-W_{\text {Fuel }}
$$

\subsection{Handling qualities}

Handling qualities involves the evaluation of the stability and control characteristics of the aircraft. According to the consideration formulated above, the optimal RFME configuration should have similar longitudinal and directional stability and control as the Do-728 aircraft. In the present study, the static margin is allowed to vary between $15.5 \%$ and $25.5 \%$ in order to stay comparable to the Do-728 configuration. These constraints have to be fulfilled by adapting the size of the horizontal and vertical tail planes and the position of the main wing.

\section{Optimisation Process}

The developed MDO process is based on a multi-level fidelity approach, where the aerodynamics is simulated using CFD methods while the weight and the handling qualities are assessed using preliminary design tools. The configuration evaluation process is implemented in the ModelCenter [6] optimisation framework. All data to define the geometry of the aircraft, its properties and flight performance are stored in the DLR common CPACS (Common Parameterized Aircraft Configuration Schema) [8] format. The complete optimisation process is presented in Figure 1. After the optimiser set the new engine and wing positions, the first step is the sizing of the tail planes. CATIA then updates the geometry followed by the mesh generation with Centaur [9] and the CFD evaluation with TAU [10]. Additionally, the angle of attack and of HTP are automatically adjusted to reach the trimmed state for a given lift. Finally, a slight change of the angle of attack is applied to compute the static margin.

It is worth to notify that the MDO process runs on three different platforms and requires quite intelligent linking between single modules to ensure correct data flow. CATIA and ModelCenter run on the Windows PC. The tail planes sizing procedure, the mesh generation, the trim of the aircraft module, the calculation of the objective and constraints and the file $\mathrm{I} / \mathrm{O}$ 
with CPACS run on the Linux workstation. The calculation of the aerodynamic performance is done on the Linux cluster with queuing system.

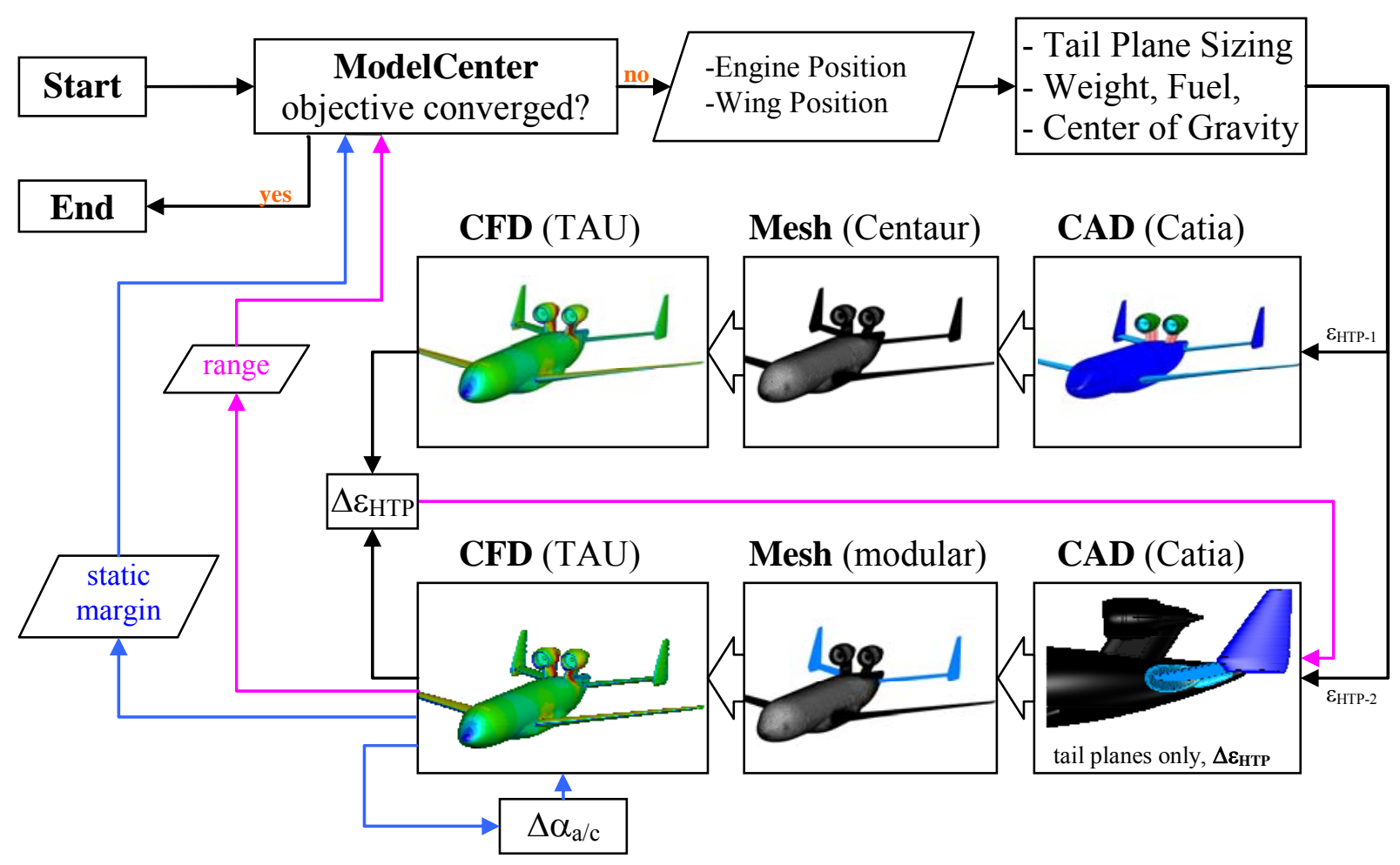

Figure 1: Optimisation process

\subsection{CAD representation and parameterisation}

The CAD geometry is defined via CATIA in a fully parameterised way and depends on the optimiser and MDO modules. The engine position is defined by three design parameters set by the optimiser: one for the longitudinal position along the fuselage axis, a second for the distance between the nacelle and the fuselage and a last one for the engine position up and down along the circumferential direction. The parameterisation is such set to avoid collision between CAD parts. The longitudinal position of the wing is controlled by the fourth design parameter.

The vertical and horizontal tail planes are sized according to the tail plane sizing module and the angle of incidence of the HTP by the trimming module. All intersections between parts are updated as well and the resulting geometry is mapped to separate layers to obtain a robust connection to the mesh generation tool.

\subsection{Handling qualities}

Two approaches are used to fulfil stability and control criteria of each aircraft configuration. The longitudinal stability is evaluated based on the CFD results and is later given as a constraint to the optimiser. The longitudinal control, and directional stability and control are ensured by correctly sizing the tail planes. This sizing process relies on preliminary design approach [11] and then calibrated on the Do 728 aircraft. The sizing of the tail planes is done iteratively, since its size depends, among other things, on the $\mathrm{CG}_{\mathrm{a} / \mathrm{c}}$ position which is affected in return by the tail planes size. 


\subsubsection{Longitudinal stability}

Static margin describes the ability of the aircraft to return into the statically stable state without pilot interaction when a small disturbance in the inflow occurs. It is defined as the distance between $\mathrm{CG}$ and the $\mathrm{AC}$ of the aircraft [4] and can be calculated as in the equation described below.

$$
S M=\frac{x_{A C}-x_{C G}}{C_{M A C}}=-\frac{d C_{M}}{d C_{L}}
$$

\subsubsection{Longitudinal control}

The go-around case with the most front CG position is the worst case in terms of control and the required HTP size can thus be calculated as follows:

$$
\frac{S_{H}}{S_{W}}=\frac{C_{L}}{C_{L, H} \cdot \eta_{H} \cdot \frac{l_{H}}{C_{M A C}}} \cdot \frac{X_{C G-A C}}{C_{M A C}}+\frac{C_{M, W}+C_{M, E}}{C_{L, H} \cdot \eta_{H} \cdot \frac{l_{H}}{C_{M A C}}}
$$

All geometrical variables such as $l_{H}, C_{M A C}, X_{C G-A C}$ are known per definition. The other variables such as $C_{M, W}$ are known from the CFD results of the Do 728 wind tunnel model, $\mathrm{C}_{M, E}$ is the product of trust and engine distance to $C G_{a / c}$ and $\eta_{H}=0,9$ is chosen as a reasonable number. The unknown $C_{L, H}$ can be calculated using known $S_{H}$ and $S_{W}$ of the Do-728 and considering this airplane as controllable.

\subsubsection{Directional control}

The "one engine off" case is the most critical situation for the VTP sizing. The VTP has to produce enough yaw moment, around the most aft $\mathrm{CG}_{\mathrm{a} / \mathrm{c}}$, in order to compensate the asymmetrical thrust. The VTP size $S_{V}$ can be calculated with following equation:

$$
\frac{S_{V}}{S_{W}}=\frac{1}{\frac{1}{2} \cdot \rho \cdot V_{M C}^{2}} \cdot \frac{N_{E}+N_{D}}{K_{V C}} \cdot \frac{1}{l_{V}}
$$

The unknown coefficient of the equation is $K_{V C}$ which depends on the VTP planform and the ruder deflection. It is calculated for given $S_{V}$ of the Do-728, at first. Since the VTP of the RFME is very similar, to the one from the Do-728, despite being shift to the HTP tip, the calculated value of $K_{V C}$ is used to size VTP of the RFME.

\subsubsection{Directional stability}

The minimal $S_{V}$ to fulfil yaw stability requirement is calculated in the similar manner as described above. The ruling equation is as following:

$$
\frac{S_{V}}{S_{W}}=K_{V, S} \cdot \frac{b_{W}}{l_{V}}, \quad \text { where } K_{V, S}=\frac{C_{N}^{\beta}-C_{N, F}^{\beta}}{-C_{Y, V}^{\beta}}
$$

The $K_{V, S}$ coefficient includes maximal accepted yaw momentum coefficients of the complete $\mathrm{a} / \mathrm{c}$, fuselage and VTP respectively. According to preliminary design calculations in [11] the largest VTP surface is required at take-off speed and for the most aft CG position.

\subsection{Calculation of Mass Components}

The introduced changes will impact the mass of the aircraft. In order to maintain the MTOW constant, it is assumed that any change of the wing, fuselage, engine and pylon masses are automatically compensated by an equivalent change of fuel mass.

Thanks to different analysis [12] based on PrADO [13], the wing and fuselage mass of the RFME configurations are known for a range of engine and wing positions. In order to use these data within an optimisation process, surrogate model for wing and fuselage mass were derived using polynomial equations. In the same way the trade-off between the size and the 
mass of the tail planes has been assessed using PrADO and then approximated using first order equations. Since the PrADO based assessment shows weak sensitivity of the pylon mass to the engine position, the calculation of the pylon mass relies on a simplified pylon box and equivalent stress theory [14]. The last defines the thickness of the pylon cross section and its mass based on the transversal and shear stress, and bending loads. These are derived from the pylon geometry for the given engine weight, blade off treatment and thrust.

\subsection{Aero Performance Prediction}

\subsubsection{Mesh procedure}

The computation of the aerodynamic coefficient is based on Euler flow around the complete aircraft. The Euler approach was here preferred to Navier-Stokes because it allows a reliable performance prediction in cruise flight at relatively low cost.

The total number of the mesh vertices is about 700'000, and may vary according to the tail planes and pylon sizes. The surface of the aircraft is discretised by about 290 '000 vertices, while $30 \%$ are used for the wing, and $14 \%$ and $11 \%$ are used for the horizontal and the vertical tail plans respectively. The mesh generation time for the complete grid takes about 20 minutes on a $2,6 \mathrm{GHz}$ AMD Opteron computer.

Since the evaluation of the aircraft aerodynamic performance in trimmed condition required the flow simulation for three different HTP incidence angles, three different meshes are required. The modular mesh approach available in CENTAUR is used to decrease the mesh generation time significantly since only a part of the mesh around the changed geometry has to be regenerated. Even though all tries done in the DLR in the past to use this functionality did not work satisfyingly, it was possible to setup a process for proper modular mesh generation. Even more, for the first time ever, thanks to the robust CAD connection to CENTAUR, it was possible to generate modular meshes in batch mode. This approach permits to reduce the mesh generation time for a new HTP orientation by a factor of three compared to classical full mesh generation.

\subsubsection{Flow solver}

The CFD solver is a DLR-TAU solver able to solve Euler as well as RANS equations on three- dimensional hybrid meshes. In the study, the spatial discretisation is done by Classic Central Jameson scheme. The scalar dissipation was chosen to stabilise the calculations. The LUSGS (Lower-Upper Symmetric Gauss-Seidel) time integration was used to accelerate the convergence. The aerodynamic coefficients are computed by the integration of pressure forces at the surfaces. The flow computation is performed on $16 \mathrm{AMD}$ cores of the $\mathrm{C}^{2} \mathrm{~A}^{2} \mathrm{~S}^{2} \mathrm{E}$ cluster and a single flow computation needs about 20 minutes.

In theory, the engine conditions have to be set such to compensate the aircraft drag. In the present scenario, the influence of engine settings onto the aerodynamic performance is neglected and it is implicitly assumed that the engine produces enough power whatever the drag is. Under these assumptions, the engine conditions are thus kept constant and identical to the Do-728 configuration.

\subsubsection{Trim and static margin calculation procedure}

The drag prediction for zero pitching moment allows taking into account the trim drag and gives thus a more accurate aerodynamic performance prediction. Assuming a linear relationship between pitching moment and the angle of incidence of the HTP $\left(\varepsilon_{H T P}\right)$ the required angle is calculated by linear interpolation based on CFD at two given $\varepsilon_{H T P}$. Figure 2 shows the pitching moment and drag coefficients over the HTP angle of incidence for two different geometries. The trimmed states could be achieved for both geometries. It can be observed that the non-linear evolution of the drag coefficients which stresses the need to perform a third calculations to get the drag more accurately. 
The calculation of the static margin relies on eq. (3) and uses pitching moment and lift coefficients from two CFD calculations. The first is the one with the achieved trimmed state at required lift. The second is the one where the angle of attack is increased by $0.2^{\circ}$ compared to the first calculation. The momentum reference point is in both cases the centre of gravity location in the most rear position which is calculated for each configuration taking into account changes of the aircraft components mass and its location.

\subsection{Optimiser}

The Design Explorer is a global search algorithm applicable for non-smooth and noisy design spaces [7]. At first it will explore the design space by conducting a design of experiment (step 1 ) in order to create a surrogate model (step 2). It will then perform a gradient based optimisation on that surrogate (step 3) and perform new analysis on the local optimums found (step 4). In case the new analysis vary a lot from the surrogate model, the latter will be reconstructed and the step 3 and are reiterated until convergence. The final step is a local pattern search.

\section{Optimisation Results}

The goal function of the optimisation was the maximisation of the mission range, as introduced previously. The engine and wing positions were the design parameters. It was assumed that all fuel after climb available can be used for cruise flight. The aerodynamic performance was calculated at beginning of cruise.

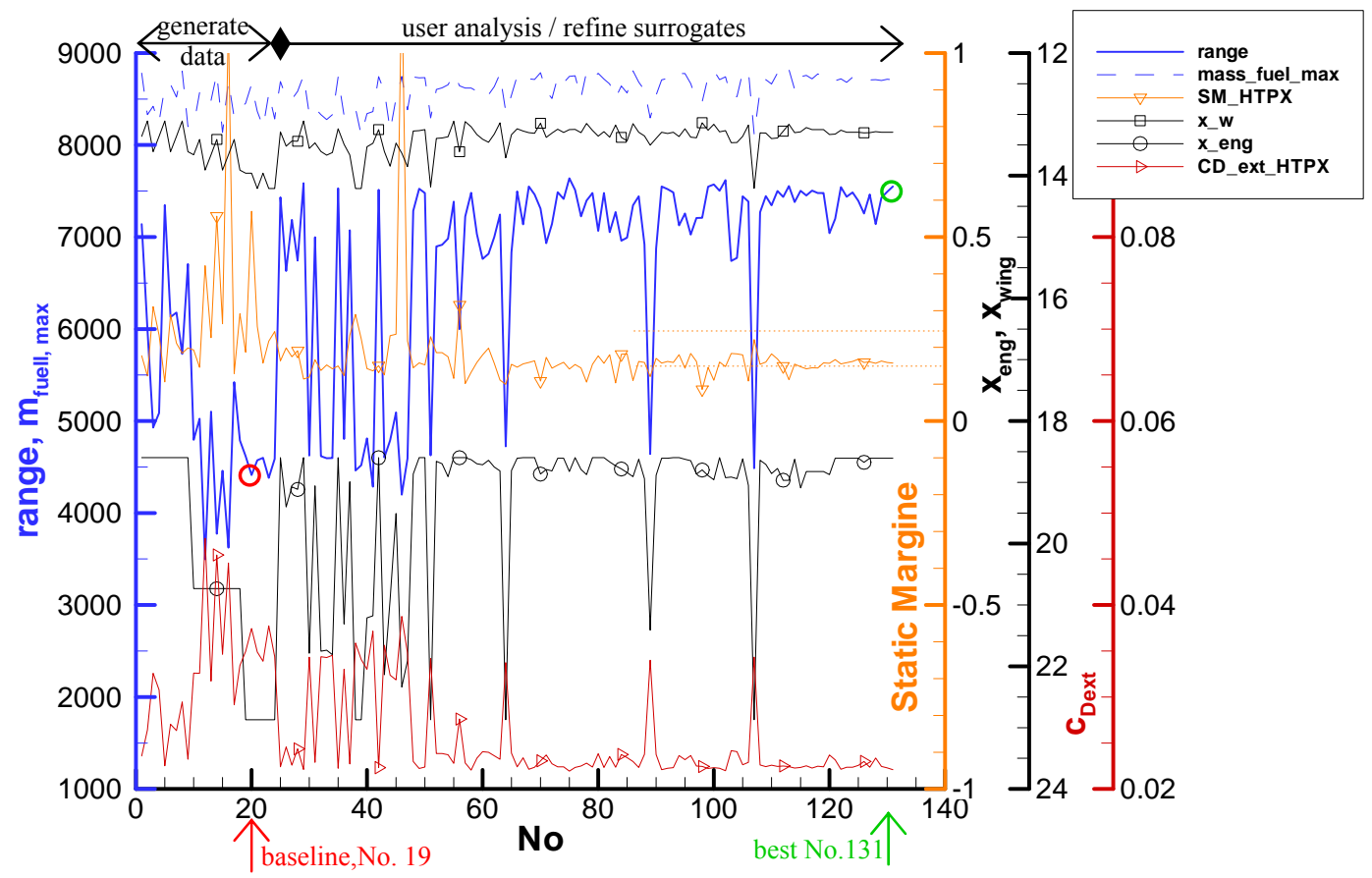

Figure 3: Optimisation history

Figure 3 shows the history of the optimisation. The optimisation resulted in nearly $64 \%$ more range after 131 cycles, including 393 grid generation and 524 CFD analyses, performed within 260 hours. The optimisation does not start with the baseline RFME geometry, due to the procedure the optimiser works. However, the geometry No. 19 is base line configuration. It has a range of $4516 \mathrm{~km}$. The best range of $7550 \mathrm{~km}$ was achieved with the geometry No. 131. Following the equation (1), the range of the Do 728 aircraft would be $6398 \mathrm{~km}$. 
Over the 170 geometries tested, only 40 failed during the analysis, 8 times due to mesh process and 32 times due to a numerical error occurring during the CFD. That means a failure rate of 5\% due CFD and $1.6 \%$ due to mesh generation, since three different meshes are used to analyse each configuration. Considering complexity of the geometry and its variation range it is still a good result.

Figure 4 shows the initial and optimised configurations. The engine of the optimised configuration is shifted forward remarkably. The wing slightly moved forward in order to provide the requested static margin, but it is still behind the wing position of the Do-728 aircraft.
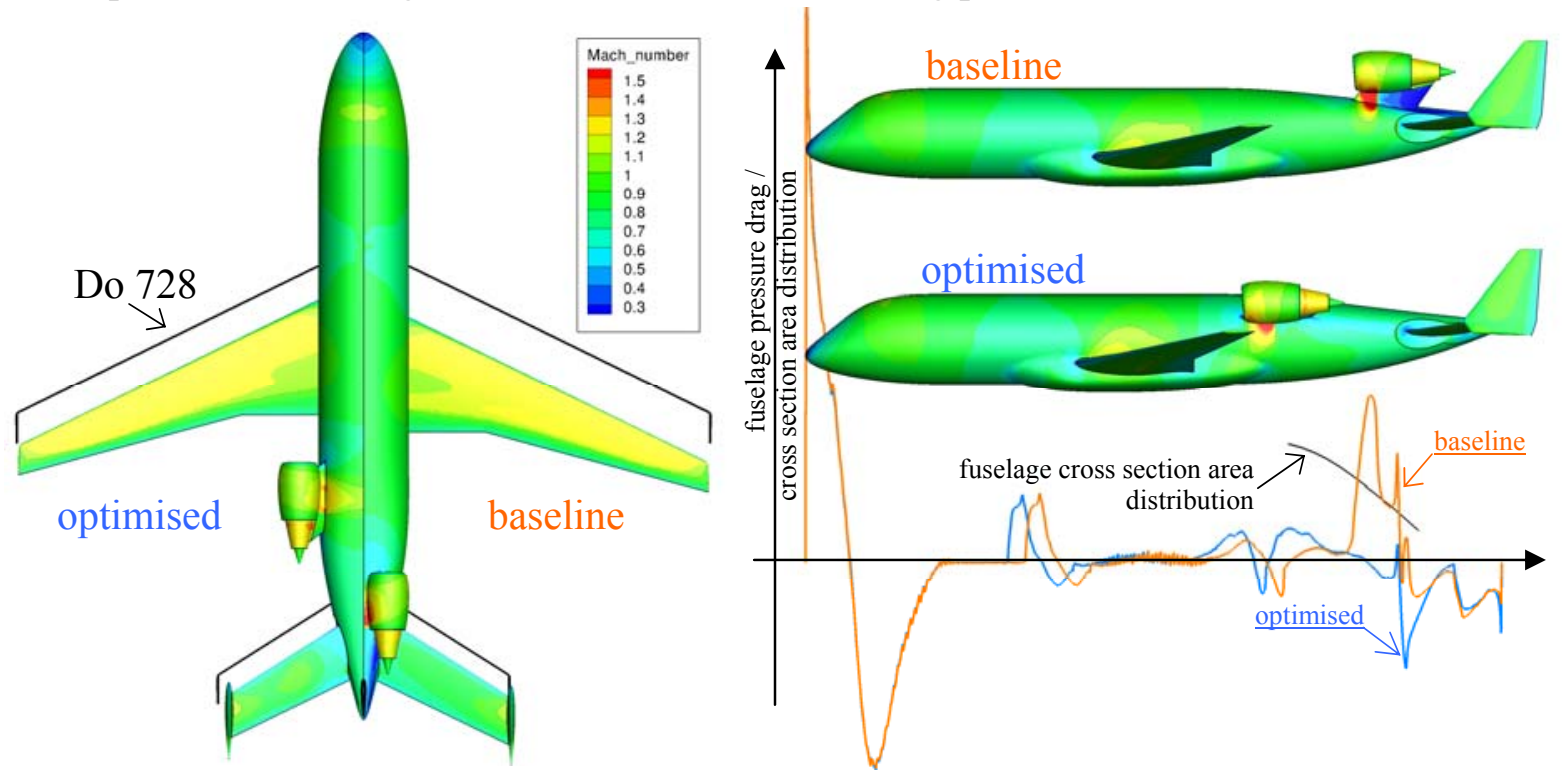

Figure 4: Comparison base line geometry vs. optimised

The increase of the mission range is achieved due to a reduction of the drag and the structural weight. The diminution in drag increases the lift to drag ratio by more than $58 \%$ and impact directly the mission range, while the weight "saved" is used as $3 \%$ of additional fuel to extend the range of the aircraft. The most of the drag minimisation is observed on the fuselage and nacelle. From the structure side, the main contributor of the weight decrease is the pylon. Since the pylon weight assessment is exclusively relying on a simplified model, its accuracy has not been demonstrated so far. Nevertheless, all other components (wing, fuselage, HTP and VTP) also contribute to the weight decrease which confirms the gain of the mission range, at least from the quality point of view.

Figure 4 and Figure 5 help to understand where the drag minimisation observed on the fuselage comes from. A certain amount of drag is observed on the fuselage close to the initial engine position. First of all, the cross section area distribution declines rapidly in that part of the fuselage. The forward engine shift moves extremely low pressure region ahead of the backward orientated part of the fuselage and reduces so the drag of the rear part of the fuselage. The second most drag minimisation is observed on the nacelle. Increased engine distance reduces

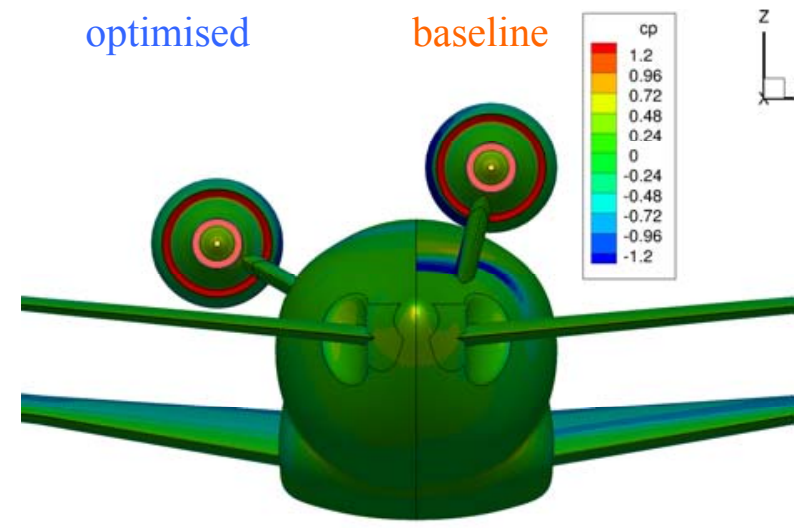

Figure 5: Pressure distribution on initial and optimised configurations, rear view the air flow acceleration along the nacelle inboard side. This leads to less lowered pressure acting on the backward orientated part of the nacelle, as Figure 5 shows, and will be here the main reason for reduced drag too. 


\section{Conclusion}

In the present study a multi-disciplinary engine integration for an aircraft with the rear fuselage mounted engines was performed. The significant increase of the flight performance with in a realistic time frame proves the efficiency and robustness of the developed optimisation process based on the multi-fidelity approach. The considerable variation of the configuration could be assessed thanks to the "CAD in loop" approach and the new "CAD to mesh creator" geometry transfer techniques. Finally the results of the optimisation are presented and the reasons for the of the flight performance increase are explained.

The future work will include the adaptation of the fuselage shape near the pylon and the pylon incidence angle in order to minimise or even to avoid the shock occurring between the pylon and the fuselage. Furthermore the optimisation process will be extended by including the noise shielding prediction.

Acknowledgments: The author would like to thank Josef Natterer for his support by the pylon mass prediction module development.

\section{References}

[1] Rossow, C.-C.; Godard,.J:-L.; Hoheisel, H.: "Investigation of Propulsion Integration Interference Effects on a Transport Aircraft Configuration”, AIAA Journal of Aircraft, Vol. 31, No. 5, pp. 1022-1030, 1994

[2] Brodersen, O. und Taupin, K. und Maury, E. und Spieweg, R. und Lieser, J. und Laban, M. und Godard, J.-L. und Vitagliano, P.L. und Bigot, P. (2005) "Aerodynamic Investigations in the European Project ROSAS", 23rd AIAA Applied Aerodynamics Conference, Toronto (can), 06.-09.06.2005 .

[3] Raymer, Daniel P.: "Aircraft design: a conceptual approach", ISBN 0-930403-51-7, 1989

[4] Torenbeek, E.: "Synthesis of subsonic airplane design", ISBN 90-298-2505-7, 1976

[5] Schlichting, H.; Truckenbrodt, E.: "Aerodynamik des Flugzeuges”, 3. Aufl., zweiter Band, ISBN 3-540-67375-X, 2001

[6] http://www.phoenix-int.com/software/phx_modelcenter.php, 2010

[7] ModelCenter documentation

[8] Liersch, C.M.; Hepperle, M.: “ A Unated Approach for Multidisciplinary Preliminary Aircraft Design.” CEAS European Air and Space Conference, Manchester, United Kingdom, 2009

[9] www.centaur-soft.com, 2010

[10] Schwamborn, D.; Gerhold, T.; Heinrich, R.: The DLR TAU-Code:

Recent Applications in Research and Industry, In proceedings of European Conference on Computational Fluid Dynamics ECCOMAS CDF 2006, Delft, The Netherlands, 2006

[11] HAW Hamburg, Skript zur Vorlesung Flugzeugentwurf, Prof. Dr.-Ing. D. Scholz, 1999

[12] Hader, C.; "Datenanalyse Fairchild Dornier 728 MDORMEC", DLR Institute of Aerodynamics and Flow Technology, 2007

[13] Werner-Westphal, C.; Heinze, W.; Horst, P.: "Multidisciplinary Integrated Preliminary Design Applied to Unconventional Aircraft Configuration", AIAA Journal of Aircraft, Vol.45, No.2, pp.581-590, 2008

[14] Grote, K.-H.; Feldhusen, J.: "Dubbel - Taschenbuch für Maschinenbau", 21. Auflage, ISBN 3-540-22142-2, 2005 